\title{
Intermédialités
}

Histoire et théorie des arts, des lettres et des techniques

Intermediality

History and Theory of the Arts, Literature and Technologies

\section{Autour du portrait d'identité : visage, empreinte digitale et ADN}

\section{Hélène Samson}

Numéro 8, automne 2006

Envisager

Facing

URI : https://id.erudit.org/iderudit/1005540ar

DOI : https://doi.org/10.7202/1005540ar

Aller au sommaire du numéro

Éditeur(s)

Centre de recherche sur l'intermédialité

ISSN

1705-8546 (imprimé)

1920-3136 (numérique)

Découvrir la revue

Citer cet article

Samson, H. (2006). Autour du portrait d'identité : visage, empreinte digitale et ADN. Intermédialités / Intermediality, (8), 67-82.

https://doi.org/10.7202/1005540ar
Résumé de l'article

La photographie du visage, l'empreinte digitale et l'autoradiogramme de l'ADN sont considérés comme différentes modalités d'un même paradigme d'identification des individus fonde sur l'inscription corporelle de l'identité et sur l'objectivité de l'enregistrement. L'auteur analyse ces présupposés et illustre leur application avec la mise au point du portrait d'identité par Alphonse Bertillon à la fin du XIXe siècle. Malgré l'évolution des modalités d'identification — du visage à l'ADN — le principe de la physiognomonie demeure, c'est-à-dire que l'indice corporel servant à contrôler l'identité sert aussi à diagnostiquer une identité essentielle. L'auteur souligne que la chirurgie, la génétique et l'image numérique, qui participent à l'évolution des techniques d'identification, modifient la conception et la représentation contemporaines du visage. 


\title{
Autour du portrait d'identité: visage, empreinte digitale et $A D N$
}

\author{
Hélène Samson
}

e portrait d'identité est une forme de représentation extrêmement fami-

- lière, puisqu'il se retrouve invariablement sur les nombreuses cartes qui donnent accès aux services publics et sur les passeports. Son vocabulaire est très limité: un gros plan photographique du visage de face, inexpressif, sur un fond neutre, auquel s'ajoute une vue de profil dans le cas de la fiche judiciaire. Sa syntaxe est rigoureusement réglée en des relations fixes entre la figure et le fond, le plan, le cadrage, la mise au point, l'éclairage et la réduction de la figure par rapport au visage de référence. La forme du portrait d'identité a été «standardisée » dans les années 1880 par Alphonse Bertillon, alors employé au Service de police de la Préfecture de Paris, pour les besoins d'un système d'identification des délinquants et des récidivistes (fig. 1). Le bertillonnage s'est vite répandu à toute l'Europe et il a été adopté par la Police canadienne et américaine dès 1887. Cependant, l'hégémonie de cette technique d'identification n'aura pas été de longue durée, puisque, en 1902, tous les services judiciaires du monde ont systématisé le recours aux empreintes digitales. Jusqu'à ce jour, aucune enquête n'en fait l'économie. Toutefois, depuis les années 1980, l'identification génétique et la biométrie numérique se sont ajoutées à la panoplie des moyens d'identification des individus. Nous voulons montrer que ces différentes techniques - le portrait photographique, l'empreinte digitale, le profil génétique et la biométrie - sont des variations de modalités sur un même paradigme: celui qui définit à l'origine le portrait d'identité. Nous ferons d'abord l'analyse des présupposés du portrait d'identité dans sa forme historique du $\mathrm{XIX}^{\mathrm{e}}$ siècle, afin de mettre en évidence le maintien de ces présupposés dans les techniques d'identification actuelles. Ensuite, nous verrons que les nouvelles conditions technologiques de la photographie et du corps entraînent des changements dans la pratique du portrait et comportent des enjeux identitaires. 


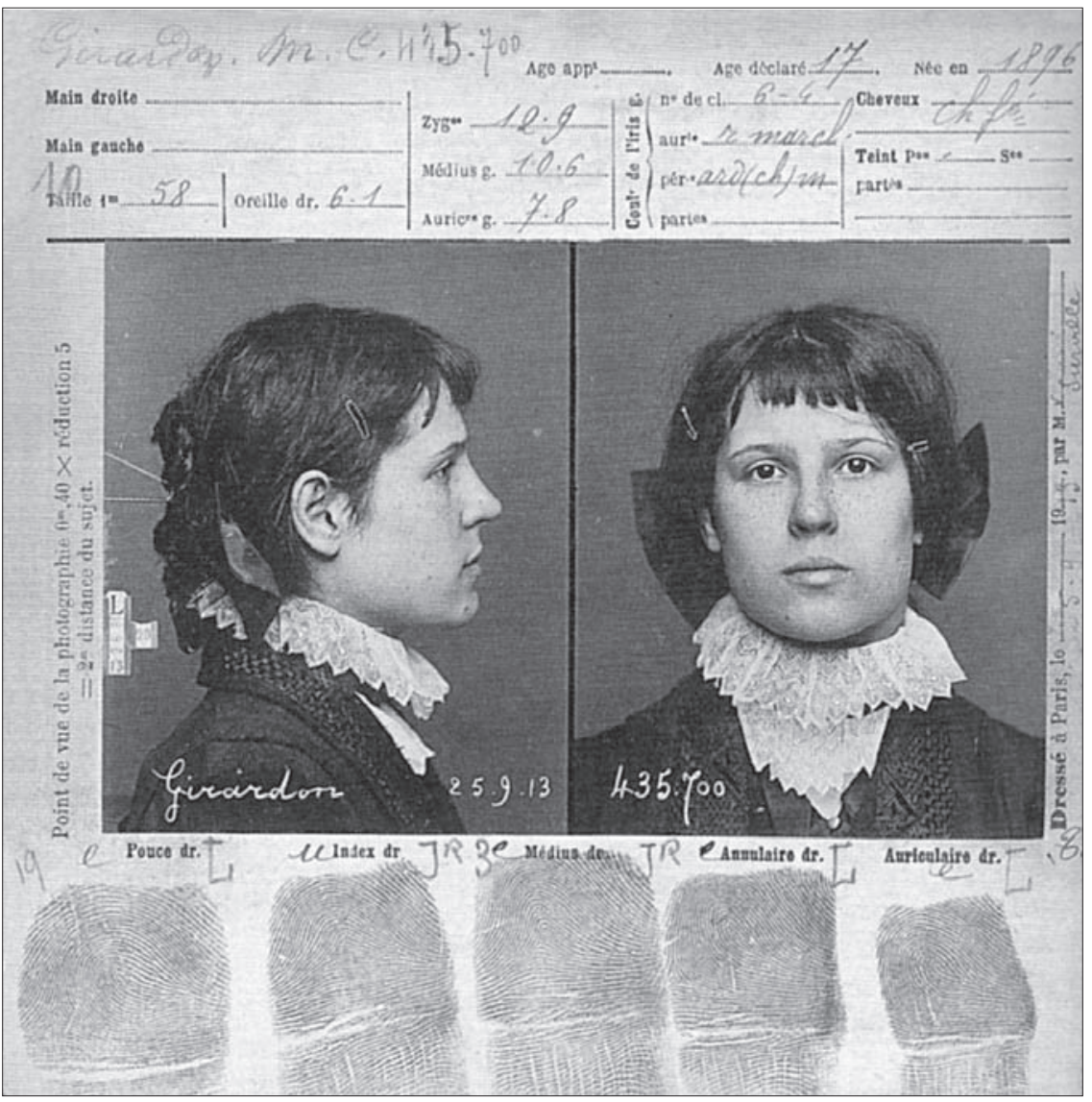

Fig. 1. Fiche signalétique de M. C. Girardon, 25 septembre 1913, Préfecture de police, Paris, photographie argentique et empreintes digitales. (C) Musée des collections historiques de la préfecture de Paris.

Les présupposés idéologiques du portrait d'identité sont l'inscription corporelle de l'individualité et l'objectivité de la représentation. En termes pragmatiques, cela signifie que cette technique d'identification nécessite la figuration d'un aspect individuel unique - en l'occurrence, le visage - et requiert que cet aspect soit enregistré de manière automatique pour en garantir la validité. En contre-exemple, un portrait au visage complètement stéréotypé, de même qu'un portrait «fait main », ne saurait remplir la fonction d'identification; l'un n'étant pas différentiel, l'autre n'étant pas objectif. Lorraine Daston et Peter Galison 
soutiennent que la notion d'objectivité est historique et qu'elle s'est cristallisée dans la seconde moitié du XIX ${ }^{\mathrm{e}}$ siècle'. Le concept est lié au caractère mécanique de l'enregistrement qui permet d'éliminer l'influence du jugement personnel dans l'observation et dans la représentation de la nature. Lobjectivité se définit donc par la négative, c'est-à-dire par l'absence d'intervention arbitraire. Daston et Galison ont analysé et historicisé cette notion en étudiant l'évolution des atlas scientifiques du XVIII ${ }^{\mathrm{e}}$ au Xx $\mathrm{Xx}^{\mathrm{e}}$ siècle. Ils ont constaté que la publication d'atlas photographiques étaient en progression à la fin du XIx ${ }^{\mathrm{e}}$ siècle. Phénomène qui participe de la formation du concept d'objectivité puisque la photographie y est présentée comme le parangon de l'objectivité. Par exemple, en 1845, Alfred Donné, médecin, fait appel à la microphotographie pour constituer l'atlas de son Cours de microscopie complémentaire des études médicales. Dans son introduction, il affirme que le nouveau procédé - le daguerréotype - représente « exactement les objets, indépendamment de toute interprétation [et qu'il prévient] l'influence des idées théoriques de l'auteur² ${ }^{2}$. Jeff Rosen, s’intéressant aussi à l'idéologie de l'objectivité, s'est penché sur un autre atlas, La photographie zoologique ou représentation des animaux rares des collections du Muséum d'Histoire naturelle, publié en 1853 sous la direction de Louis Pierre Rousseau et de JeanMarie Devéria. Il considère que la naturalisation des figures dans la Photographie zoologique s'appuie sur trois conditions d'objectivité fournies par la photographie: l'appareil, qui offre une fidélité optique et un automatisme de l'enregistrement; le cadrage, qui définit l'objet selon le concept de spécimen - notion déjà courante dans les cabinets de curiosité et dans les musées d'histoire naturelle et enfin la reproduction et la diffusion massive de clichés "purement photographiques ", c'est-à-dire non retouchés 3 . La valorisation scientifique de la photographie s'est effectuée aussi en astronomie, où l'acuité visuelle possède une valeur heuristi$q^{4}{ }^{4}$. L'archéologie est un autre domaine de formation du concept d'objectivité photographique, notamment avec la mission d'Auguste Salzmann à Jérusalem de

1. Lorraine Daston et Peter Galison, «The Image of Objectivity ", Representation, $\mathrm{n}^{\circ}$ 40, automne 1992, p. 81-128.

2. Alfred Donné, «Atlas. Introduction», dans André Rouillé, «L'euphorie des scientifiques (1844-1853)», La photographie en France, Paris, Macula, 1989, p. 73.

3. Jeff Rosen, «Naming and Framing "Nature" in La photographie zoologique", Word \& Image, vol. 13, n 4, oct.-déc. 1997, p. 377-391.

4. Les premiers enregistrements photographiques du soleil se font sous l'instigation de François Arago, en France, et de Sir John Herschel, en Angleterre, dans les années 1845 et 1847. À ce sujet, on consultera Ann Thomas, «Capturing Light: Photographing the Universe », dans Ann Thomas (dir.), Beauty of Another Order. Photography and Science, New Haven, Ottawa, Yale University Press, Musée des beaux-arts du Canada, 1997, p. 197-200. 
1852 à $1854^{5}$. Il s'agissait pour Salzmann de prouver par photographies les hypothèses de l'archéologue Ferdinand de Saulcy. Selon Abigail Solomon-Godeau, la photographie de Salzmann, typique des missions photographiques françaises du Second Empire, constitue «[...] an act of scientific documentation and objective reporting to be rendered with the impartial and truth-telling eye of the camera ${ }^{\star}$. Ces premières applications scientifiques de la photographie, soutenues par l'État et par les institutions du savoir, sont des jalons dans la formation du concept d'objectivité. Elles ont permis de légitimer l'usage scientifique de la photographie dans les travaux de Cesare Lombroso et de Francis Galton, vers 1875-1878, sur la typologie du visage humain. Mais surtout, cette objectivité de la photographie a été l'un des postulats de l'identification par le portrait photographique dans l'anthropométrie signalétique de Bertillon.

L'inscription corporelle de l'identité constitue le second présupposé fondamental du portrait d'identité. La médecine a joué un rôle important dans l'établissement de ce postulat. Michel Foucault a soutenu, dans La naissance de la clinique, que la médecine connaissait au XviII ${ }^{\mathrm{e}}$ siècle des mutations majeures, dont l'une a été d'identifier l'expérience clinique à celle du regard. Dans la médecine moderne, l'interprétation des signes en tant que symptômes de la maladie équivaut à «lire les structures profondes de la visibilité7». Foucault a également montré comment l'étude anatomique du cadavre a façonné une «médecine de l'individu », exercée en contact direct avec le malade, et caractérisée par l'association du visuel et du factuel. Cette association cruciale, consolidée par l'usage de la photographie, est à la base de l'identification des individus par des traits corporels visibles, et de la catégorisation et de la classification de ces mêmes individus. Cependant, il est rare que l'identification soit simplement nominale et sans préjugé, car la tradition physiognomonique vient teinter tout signe corporel d'une connotation identitaire profonde. C'est-à-dire que la croyance en l'idée que les marques du corps recèlent des informations sur la nature plus profonde des individus - le principe de la physiognomonie - a trouvé dans la photographie du visage et du corps un moyen sans précédent de s'affirmer. Les domaines de

5. L'œuvre de Salzmann a été étudiée par Abigail Solomon-Godeau, «A Photographer in Jerusalem, 1855: Auguste Salzmann and his Time», Photography at the Dock. Essays on Photographic History, Institutions and Practices, Minneapolis, University of Minnesota Press, 1991, p. 150-168; le cas est documenté par André Rouillé, dans «Controverse archéologique: la preuve par la photographie (1855-1861)», La photographie en France, p. 136-143.

6. Abigail Solomon-Godeau, «A Photographer in Jerusalem», p. 154.

7. Michel Foucault, La naissance de la clinique, Paris, Presses universitaires de France, 1963, p. 89 . 
l'ethnologie et de l'anthropologie à la période coloniale abondent d'exemples à ce sujet ${ }^{8}$.

L'histoire du portrait d'identité illustre l'application de ces présupposés. Plusieurs disciplines y ont contribué par des propositions fondées sur l'enregistrement photographique des traits du visage. Cependant, l'approche scientifique du portrait s'est amorcée dans une perspective typologique, c'est-à-dire de généralisation. Ensuite est venue la recherche de différenciation individuelle avec le portrait d'identité en réponse aux besoins de l'enquête judiciaire. En psychiatrie, les travaux photographiques des docteurs Hugh Welch Diamond, directeur de l'asile du Surrey, et Jean-Martin Charcot à la Salpêtrièrie, se situent dans la tradition des atlas scientifiques. Ce sont essentiellement des travaux d'illustration et de catalogage des plus «remarquables cas» de maladies mentales. Pour ces médecins, la photographie s'est imposée comme le moyen de réaliser cette tâche: «Photography gives permanence to these remarkable cases [...] and makes them observable not only now but for ever, and it presents also a perfect and faithful record [...]» écrivait Diamond dans son allocution à la Royal Society sur l'application de la photographie à la physiognomonie des maladies mentales ${ }^{9}$. L'éminent professeur Charcot, quelque trente ans plus tard, étayant de photographies ses leçons sur l'hystérie, renchérissait: "Voilà la vérité. [...] Vous savez que j’ai pour principe de ne pas tenir compte de la théorie et de laisser de côté tous les préjugés [...] je ne

8. Plusieurs essais et catalogues d'expositions ont été consacrés au rapport entre l'anthropologie et la photographie au XIx ${ }^{\mathrm{e}}$ siècle. Pour n'en citer que quelques-uns: Kathleen Stewart Howe, First Seen, Portraits of the World's Peoples, Santa Barbara, California, Santa Barbara Museum of Art, 2004; Nicholas Mirzoeff, «Photography at the Heart of Darkness », Bodyscape, New York, Londres, Routledge Press, 1995; Ruth Charity, Christopher Pinney et al. (dirs.), The Impossible Science of Being: Dialogues Between Anthropology and Photography, Londres, The Photographer's Gallery, 1995; Elizabeth Edwards (dir.), Anthropology and Photography 1860-1920, New Haven, Londres, Yale University Press, The Royal Anthropological Institute, 1992; Benoît Coutancier, «Peaux-Rouges». Autour de la collection anthropologique du Prince Roland Bonaparte, Paris, Éditions de l’Albaron, Photothèque du Musée de l'Homme, 1992; Melissa Banta et Curtis M. Hinsley (dirs.), From Site to Sight. Anthropology, Photography, and the Power of Imagery, Cambridge, Massachusetts, Peabody Museum Press, 1986.

9. Hugh Welch Diamond, «On the Application of Photography to the Physiognomics and Mental Phenomena of Insanity » (vers 1858), texte d'allocution publié dans Journal of Photographic Society, $n^{o s}$ 3-4, 1856-58, et commenté par Martin Kemp, "A perfect and Faithful Record": Mind and Body in Medical Photography Before 1900 ", dans Ann Thomas (dir.), Beauty of Another Order, p. 120-149, et par Sander Gilman, The Face of Madness, Hugh W. Diamond and the Origin of Psychiatric Photography, New York, Brunner, Mazel, 1976, p. 17-24. 
suis absolument là que le photographe; j’inscris ce que je vois ${ }^{10}[. .]$.$» . À ces mots,$ il est clair, d'une part, que les deux médecins endossaient entièrement le nouveau credo inspiré par l'enregistrement mécanique de la photographie - l'objectivité; d'autre part, que leur observation scrupuleuse de l'expression faciale, des postures, des tensions et des contractions musculaires sous-tendent l'idée d'une manifestation de la maladie mentale en surface du corps.

Au XIX ${ }^{\mathrm{e}}$ siècle, deux nouveaux courants idéologiques ont influencé les sciences sociales: le scientisme, favorisé par la philosophie positiviste d'Auguste Comte et l'eugénisme, dérivé du modèle de sélection naturelle proposée par Charles Darwin pour expliquer l'évolution des espèces. Conformément à ces courants idéologiques, le programme de recherche en sciences sociales a été marqué à la fois par une exigence méthodologique et par la prépondérance des notions d'individu, d'hérédité et de dégénérescence. De plus, le développement des statistiques durant cette même période a inspiré les protocoles de recherches de Lombroso, de Galton et de Bertillon" ${ }^{11}$. Lanthropologie criminelle de Cesare Lombroso, associée à l'école italienne, correspond à une physiognomonie de la criminalité. Selon sa théorie de l'atavisme, qui postule des vestiges de pulsions animales chez l'humain, Lombroso soutenait que le criminel-né portait sur son visage les «stigmates physiques» de la bestialité. Son ouvrage majeur paru en 1876, Luomo delinquente, contient des centaines de portraits photographiques de criminels et de déviants classés par sexe, par nationalité et présentés en tableaux. L'accumulation d'un grand nombre de cas devait permettre de révéler les «traits accusateurs » et de généraliser le faciès du criminel. Francis Galton, le père de l'eugénisme, s'est appliqué aussi à l'élaboration d'une typologie des visages. Il défendait l'idée que la transmission des attributs héréditaires - le génie au même titre que les tares - devait être contrôlée par la société. Non seulement préconisait-il de contenir les populations dégénérées en des lieux sûrs, mais aussi de les empêcher de se reproduire ${ }^{12}$. Sa méthode photographique dite du portrait composite consiste à condenser plusieurs visages en un seul par l'accumulation de six à douze expositions successives sur la plaque sensible. «Les différences sont donc dissoutes dans une opération optique de superposition, cette soustraction [...] ne conserve que les traits dominants et constants, considérés comme seuls

10. Jean-Martin Charcot [1887-1888], cité par George Didi-Huberman, Invention de l'hystérie. Charcot et l'iconographie de la Salpêtrière, Paris, Macula, 1982, p. 32.

11. Au sujet de l'influence des statistiques sur l'usage de la photographie, voir Allan Sekula, «The Body and the Archive», Richard Bolton (dir.), The Contest of Meaning: Critical Histories of Photography, Cambridge, Massachusetts, MIT Press, 1989, p. 348-359.

12. Voir Peter Hamilton, "Policing the Face», dans Peter Hamilton et Roger Hargreaves, The Beautiful and the Damned. The Creation of Identity in Nineteenth Century Photography, Londres, The National Portrait Gallery, 2001, p. 93-100. 
signifiants $^{13}[\ldots]$ ». Galton a conçu ce procédé comme un instrument de recherche scientifique pour cerner le type physionomique de toute catégorie d'individus, à commencer par le criminel, ensuite le Juif, l'Irlandais, le tuberculeux, de même que l'officier de l'armée britannique et l'ingénieur royal.

Latlas photographique de Lombroso et le portrait composite de Galton ont pour objet le type essentiel permettant, chez l'un, de reconnaître la physionomie atavique des criminels et des prostituées; chez l'autre, de révéler les traits héréditaires de certains groupes d'individus et de familles. Bien que le fondement de leur typologie s'appuie sur l'inscription corporelle de l'identité, il ne s'agit pas encore de portraits d'identité, car l'accent porte sur l'uniformité des visages. En revanche, à la même époque, les fiches que s'échangeaient criminologues et policiers dans l'exercice de leurs fonctions pour identifier les délinquants devaient montrer les traits différentiels des individus. C'est dans l'anthropométrie judiciaire, développée par Alphonse Bertillon, que la syntaxe du portrait d'identité atteint son point d'orgue. Alphonse Bertillon entre au service de la police parisienne en 1878 et découvre un registre abondant de portraits et de marques corporelles distinctives pratiquement inutilisables en raison du manque d'uniformité des données et, surtout, en raison du manque de classification adéquate. L'efficacité policière requérait de pouvoir identifier un récidiviste dans les plus brefs délais, c'est-à-dire de retrouver rapidement la fiche signalétique correspondant à la description du contrevenant. Bertillon résolut d'abord le problème du signalement. Il avait été à même de constater, à l'occasion de bavures, que la ressemblance photographique pouvait être trompeuse, sans compter que déguisements, blessures, chirurgies pouvaient rendre un individu méconnaissable. C'est pourquoi il sélectionna un indice individuel extrêmement stable et infalsifiable: l'ossature. En cela réside, comme le souligne Christian Phéline, la «véritable invention » de Bertillon ${ }^{14}$. De plus, les mesures osseuses permettaient d'enregistrer la description corporelle en termes numériques, c'est-à-dire de produire une codification de l'identité. Dès l'année suivant son arrivée, Bertillon proposa donc un système d'anthropométrie en trois parties : 1) une série des mesures corporelles, onze en tout, comprenant la taille, les dimensions des membres, des doigts, des pieds, des oreilles, de la mâchoire et du crâne; 2) une description physique de l'individu dans un vocabulaire morphologique très précis et suivant un relevé des détails corporels désignés - le front, le nez, l'oreille, l’iris, incluant les marques distinctives comme les cicatrices et les tatouges; 3 ) un double portrait photographique de face et de profil réalisé selon des règles strictes. Tous ces

13. Michel Frizot, "Corps et délits. Une ethnophotographie des différences", Nouvelle histoire de la photographie, Paris, Bordas, Adam Biro, 1995, p. 267.

14. Christian Phéline, «L'image accusatrice», Les cahiers de la photographie, $\mathrm{n}^{\circ} 17$, Laplume, ACCP, 1985, p. 10. 
renseignements se trouvaient réunis sur une fiche, y compris le portrait photographique. Bertillon résolut ensuite le problème majeur de l'archivage de ces fiches en un système qui servira de modèle pour la classification des empreintes digitales. Notons, au passage, que ce problème resurgit actuellement avec la classification des données biométriques informatisées. Dix ans après l'instauration du bertillonnage à Paris, on peut lire dans le manuel traduit à l'intention des policiers américains: "[...] the potential was to fix the human personality, to give each human being an identity, an individuality, certain, durable, invincible, always recognizable, and always capable of being proven ${ }^{15}[. .$.$] ». Des mots qui traduisent$ un désir de certitude, une adhésion totale au paradigme d'une identité définie par l'objectivité et par l'inscription corporelle de l'identité.

Durant ces mêmes années, un autre indice corporel attire l'attention: les dermatoglyphes — ou plus exactement les «dactyloglyphes » — à l'origine de l'empreinte digitale. Cette technique d'identification, plus efficace, plus facile et plus économique que le portrait photographique et l'anthropométrie combinés, détrônera le bertillonnage. Cependant, malgré cette dévaluation, on ne cessera pas de photographier les détenus selon le modèle institué à la Préfecture de Paris, mais le rôle du portrait d'identité dans l'enquête policière ne sera plus capital. En revanche, dans le milieu civil, ce petit portrait connaîtra une diffusion extraordinaire au cours du $\mathrm{xx}^{\mathrm{e}}$ siècle, sur le passeport notamment. L'histoire du passeport révèle un curieux revirement de situation: pendant des siècles, faisant office de sauf-conduit, le document désignait le voyageur, l'étranger, l'individu en situation hors-norme ${ }^{16}$. Il était un document d'exception susceptible d'éveiller le soupçon à l'égard de son porteur. Paradoxalement, au $\mathrm{xx}^{\mathrm{e}}$ siècle, le passeport, comme la carte d'identité, atteste une légitimité. C'est désormais la situation d'être «sanspapiers » qui est suspecte. De plus, les usages du portrait d'identité sont à l'origine d'une double rhétorique. Christian Phéline constate une rhétorique de «l'image accusatrice ${ }^{17}$ » liée au contexte d'usage en psychiatrie, en criminologie, et dans l'investigation policière. Par ailleurs, en se banalisant sur les cartes d'accès aux

15. Gallus Muller, «Preface», Alphonse Bertillon's Instructions for Taking Descriptions for the Identification of Criminal and Others by the Means of Anthropometric Indications, 1889, New York, AMS Press, 1977, n. p.

16. Gérard Noirel, «Surveiller les déplacements ou identifier les personnes? », Genèses. Sciences sociales et histoire, Paris, $\mathrm{n}^{\circ}$ 30, mars 1998, p. 77-100.

17. L'expression est attribuée à Ernest Lacan qui écrivait au sujet de l'usage judiciaire de la photographie: «Quel repris de justice pourrait échapper à la vigilance de la justice? Qu'il s'échappe des murs où le retient le châtiment; qu'une fois libéré, il rompe le ban qui lui prescrit une résidence, son portrait est entre les mains de l'autorité; il ne peut échapper : lui-même sera forcé de se reconnaître dans cette image accusatrice", Ernest Lacan, La Lumière, 1856, cité par Christian Phéline, «L'image accusatrice», p. 19. 
services publics, le portrait d'identité est devenu une figure sans ressemblance, au sens idéal de ce terme. Sa rhétorique est alors celle de l'anonymat, comme le suggère la photographie de Jana Sterbak, Generic Man (1987), où l'on voit un individu marqué à la nuque d'un code barre (fig. 2).

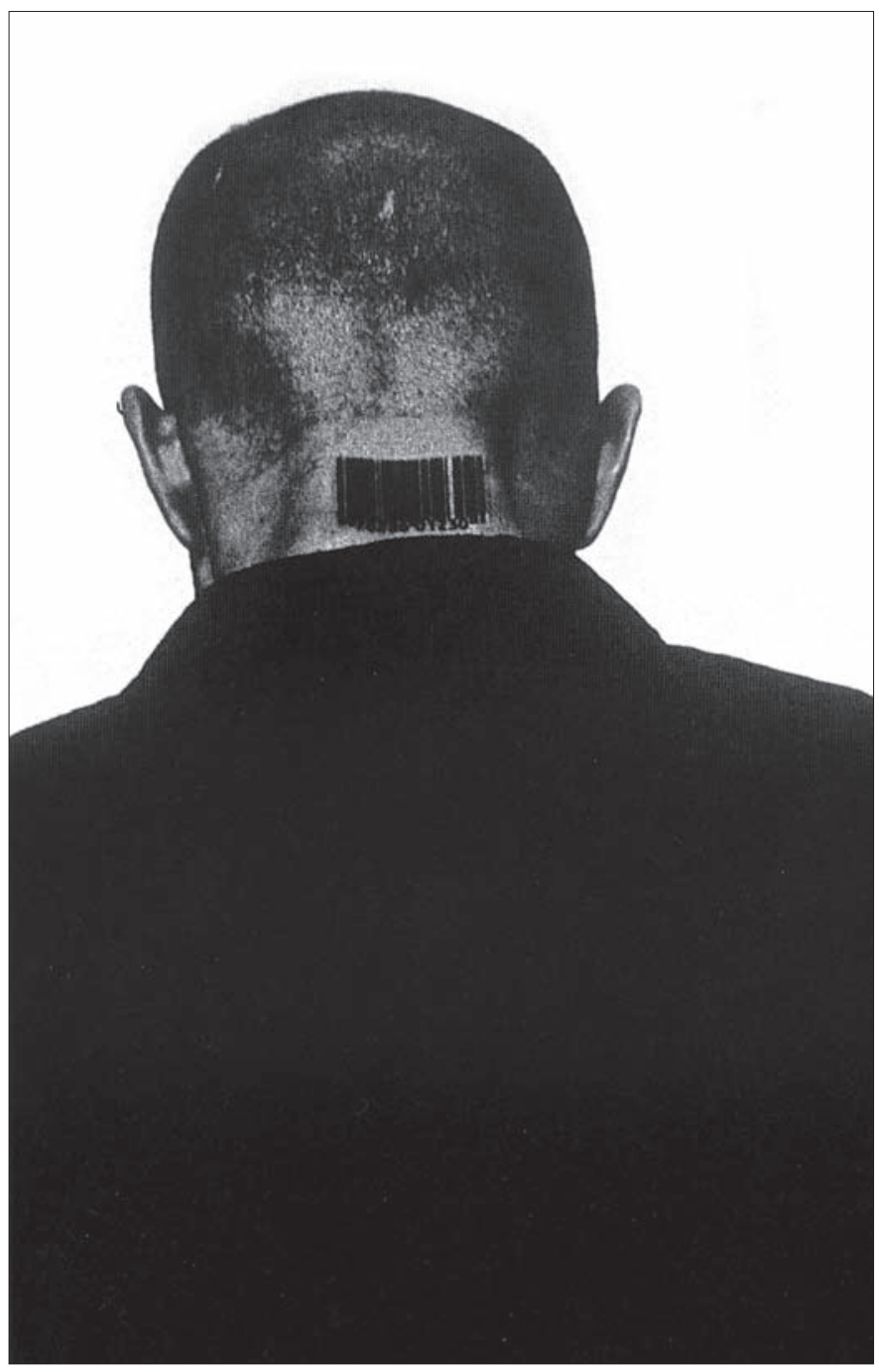

Fig. 2. Jana Sterbak, Generic Man, 1987. (c) Jana Sterbak. Avec l'aimable autorisation de l'artiste. 
En 1902, à la suite de résolutions d'enquêtes apportées par les empreintes digitales, la supériorité de ce système s'est imposée ${ }^{18}$. Malgré ce changement de modalité, il ressort que l'identification par les dermatoglyphes s'appuie sur les mêmes présupposés que le portrait photographique : l'inscription corporelle de l'identité et l'objectivité de l'enregistrement. L'objectivité est garantie par le procédé automatique de l'empreinte, lequel correspond, comme la photographie, à la sémiologie de l'indice chez Peirce. De plus, l'implantation de cette technique reproduit les mêmes problèmes - de classification, notamment - et les mêmes enjeux sociaux et philosophiques que le bertillonnage. Par ailleurs, le portrait et l'empreinte digitale ont accru l'efficacité de la surveillance dans le temps et dans l'espace. Ces deux techniques ont aussi constitué une attestation (naïve) du déterminisme biologique et ont favorisé le maintien de la physiognomonie. À ce propos, l'identification des criminels au moyen des empreintes digitales s'est accompagnée de l'ambition de faire de cet indice un outil diagnostic, c'està-dire d'étendre l'identification des individus à la détermination de leur identité. Francis Galton, en étudiant l'hérédité des dermatoglyphes, espérait déceler l'ascendance des individus, qu'il s'agisse de la race, de l'ethnie ou de la prédisposition à la criminalité. Selon une simplification de la théorie de l'évolution des espèces, il recherchait la présence de dermatoglyphes simiesques chez les individus « dégénérés ». Il ne parvint pas à soutenir cette hypothèse. Quoi qu'il en soit, les ambitions de Galton manifestent un entêtement à déterminer plusieurs caractéristiques d'un individu à l'aide d'un seul indice corporel, c'est-à-dire un désir de diagnostiquer l'identité. Aujourd'hui, l'empreinte digitale est toujours en vigueur dans l'identification des contrevenants, mais elle est dissociée de l'ambition diagnostique, même si cette possibilité est réelle ${ }^{19}$. En revanche, l'identification par le profil génétique, la troisième variation sur un même paradigme, semble indissociable de son potentiel d'informations sur l'ascendance et l'avenir biologique des individus, et ce, au-delà de leur propre connaissance d'eux-mêmes.

L'acide désoxyribonucléique (ADN) est une caractéristique individuelle comme les dermatoglyphes et le visage. Son enregistrement, sous la forme de

18. Nous renvoyons au cas «Will West» relaté par Simon A. Cole, dans Suspect Identities. A History of Fingerprinting and Criminal Identification, Cambridge, Massachusetts, Harvard University Press, 2002, p. 141-142. Ce cas relate l'existence de deux individus du même nom, dont les portraits étaient presque identiques. L'empreinte digitale permit d'attribuer à chacun sa fiche signalétique respective.

19. Au sujet des possibilités de diagnostic par l'empreinte digitale, voir Simon A. Cole, Suspect Identities, p. 307. 
l'autoradiogramme ${ }^{20}$, relève d'une technique analogue à la photographie, à la différence près que la trace est produite par la radioactivité plutôt que par la lumière. Lanalyse biochimique de la molécule d'ADN, à l'origine du profil génétique individuel, a permis de résoudre des énigmes judiciaires, notamment les crimes à caractère sexuel pour lesquels le visage et l'empreinte digitale n'étaient d'aucune utilité. Selon la théorie génétique de l'hérédité, il y aurait un lien causal essentiel entre les gènes et l'apparition des traits héréditaires. C'est en ce sens que sont interprétées les corrélations démontrées entre un gène et un phénomène biologique, d'où l'expression « code génétique » qui reconduit la métaphore linguistique. Cependant, la génétique de pointe réfute ce rapport de causalité directe entre le génotype et le phénotype, pour la simple raison qu'une corrélation n'implique pas un lien causal et que plusieurs autres facteurs entrent en jeu dans l'apparition d'un phénomène biologique, ne serait-ce que l'alimentation et l'environnement ${ }^{21}$. De plus, il s'avère qu'une très grande proportion d'ADN n'est corrélée à aucun trait ou phénomène biologique particulier ${ }^{22}$. Néanmoins, la notion d'identité génétique frappe les esprits car le discours de vulgarisation véhicule le mythe de la connaissance physiognomonique parachevée, la perspective de l'eugénisme, du clonage et de l'éradication des maladies héréditaires et dégénératives. Même si de nouvelles théories de l'hérédité viennent tempérer ces enthousiasmes, il demeure que dans l'investigation judiciaire, le profil génétique constitue le test ultime dans un grand nombre de cas, et cet usage ne peut qu'être facilité par l'informatisation de la technique et la miniaturisation des laboratoires. En ce qui concerne l'identité personnelle, l'identification génétique de l'individu, de même que toute forme d'identification corporelle objective, est déstabilisante, aversive même, car elle procure un sentiment d'aliénation. L'individu ne contrôle pas et ne comprend pas cette information qui le caractérise. Surtout, il ne peut se reconnaître dans des notations biochimiques, bien qu'il doive admettre d'un point de vue scientifique que l'indice génétique recèle des informations très exactes sur ses prédispositions biologiques.

En réponse aux besoins accrus de sécurité, dont il convient d'ailleurs de considérer la part d'inflation idéologique, la biométrie électronique (biometrics) s’avère plus adéquate que le profil génétique. L’identification par détection

20. Pour une analyse sémiologique de l'autoradiogramme, voir Hans-Jörg Rheinberger, «Auto-Radio-Graphics», dans Bruno Latour et Peter Weibel (dirs.), Iconoclash, Karlsruhe, Cambridge, ZKM, MIT Press, 2002, p. 516-519.

21. Jean-Jacques Kupiec, Pierre Sonigo, «L'erreur génétique », dans Ni Dieu ni gène. Pour une autre théorie de l'hérédité, Paris, Éditions du Seuil, 20oo, p. 76-80.

22. À ce sujet voir Thierry Bardini, «Variations sur l'insignifiant génétique: les métaphores du (non) code », Intermédialités, n 3, printemps 2004, «Devenir-Bergson », p. 163-186. 
électronique d'un indice corporel est en plein essor, qu'il s'agisse de détecter les dimensions de la main, le timbre de la voix, le réseau veineux au dos de la main ou le cône de l'iris. Ces indices corporels peuvent être enregistrés efficacement grâce à des instruments très perfectionnés. Une fois ces indices numérisés et sauvegardés dans un système de détection, la reconnaissance de l'individu peut s'effectuer automatiquement. L'application de la biométrie vise entre autres à contrôler le passage des frontières et l'accès à des sites réservés - centres récréatifs, bibliothèques, institutions bancaires, aéroports et surtout les banques de données informatiques. Les indices sont sélectionnés pour leur potentiel de différenciation, leur accessibilité et leur «infalsifiabilité». Selon ce dernier critère, tout indice est abandonné dès qu'il peut être modifié par la chirurgie plastique ou par la génétique. De plus, ces indices ne sont pas connotés - pas encore - de la possibilité de déterminer «en profondeur» l'identité de l'individu, comme c'est le cas pour le visage et le profil génétique. D’ailleurs, le discours de la biométrie, sensible aux problèmes de discrimination, insiste sur le fait qu'elle ne vise strictement que la vérification de l'identité.

L'enregistrement électronique de la voix ou de l'iris constitue une autre forme du «paradigme du portrait d'identité» - comme la photographie du visage, l'empreinte digitale et le profil génétique. Bien que la modalité de l'identification soit nouvelle, le corps demeure soumis aux principes de l'objectivité et de l'inscription corporelle de l'identité. Cependant, la biométrie présente une particularité, à savoir qu'il y a dissolution de l'artefact intermédiaire entre l'agent de contrôle et l'individu contrôlé. Autrement dit, les notations, les papiers d'identité, le NIP ou le mot de passe ne sont plus utiles. L'objet de médiation disparaît. Avec l'enregistrement de l'iris, par exemple, il suffit à l'individu de fixer un point précis quelques fractions de seconde pour accéder ou non à un site protégé. «Biometrics are turning the human body into a universal ID card of the future ", écrit Irma Van der Ploeg ${ }^{23}$. Le corps devient directement lisible par le système électronique, luimême dissimulé dans l'environnement. Van der Ploeg considère que la biométrie produira une «identité digitale» associée par conditionnement aux activités sociales sous surveillance, comme les déplacements. On peut en effet envisager que tout individu intériorise son propre circuit dans l'espace public, avec ses passages libres, autorisés, facilités ou interdits, comme un aspect de soi.

Le paradigme du portrait d'identité, qui traverse les $\mathrm{XIX}^{\mathrm{e}}$ et $\mathrm{XX}^{\mathrm{e}}$ siècles, est emblématique de la modernité dans l'objectivation et l'ordonnancement des êtres humains, de même que dans l'obsession à scruter et à lire les surfaces corporelles en quête d'une signification intérieure. Les grands portraits de Thomas Ruff

23. Irma van der Ploeg, «Written on the Body: Biometrics and Identity», Computers and Society, vol. 29, n 1, mars 1999, p. 37-44. 
réalisés dans les années 1980 reviennent sur ce paradigme moderne du portrait d'identité. Ils en sont une stylisation pour mieux remettre en question ses présupposés. Au demeurant, si ce paradigme crée de nouveaux phénomènes comme l'identité génétique et l'identité digitale, les nouvelles technologies, qui permettent cette évolution, changent la pratique du portrait. En effet, avec la numérisation de l'image, toute photographie est désormais suspecte et ne peut plus jouir de son aura d'objectivité; avec les progrès de la chirurgie, le visage - et par extension tout le corps - devient une surface de manipulation. Du point de vue judiciaire, le visage est un indice hautement falsifiable, et du point de vue social, il est une surface d'inscription des désirs d'une communauté. À ce chapitre, d'ailleurs, s'ajoute maintenant la possibilité de greffer un nouveau visage. En novembre 2005, un médecin français réussissait la première greffe partielle du visage - nez, bouche et menton. Succès qui a été répété en avril 2006 par une équipe chinoise ${ }^{24}$. Il s'agissait dans les deux cas d'individus défigurés par une morsure d'animal: de chien et d'ours. L'intervention consiste à mouler une nouvelle peau provenant d'un donneur sur les os et les muscles du receveur en rétablissant les connexions nerveuses et sanguines. Le nouveau visage est donc la combinaison des visages de l'un et de l'autre, rien de moins qu'un portrait composite vivant. Plusieurs médecins autour du monde sont en lice pour réaliser une greffe complète du visage. Les équipes de Cleveland et de Londres sont prêtes et cette dernière n'attend que l'autorisation par un comité d'éthique de procéder à l'intervention sur une victime de grandes brûlures. En regard de ses possibilités, la chirurgie plastique - comme le génie génétique - instaure une nouvelle politique du corps et par conséquent, un profond bouleversement dans la phénoménologie de l'identité personnelle. Si l'on compare l'histoire du portrait scientifique, qui a permis de nommer, de collectionner et de classer les visages comme une donnée fixe, et les conditions actuelles du portrait photographique, qui rétablissent la subjectivité et la créativité du portrait pictural, on constate qu'après avoir été dévisagé par la science - au sens de dépersonnaliser - l'individu retrouve la possibilité de s'envisager lui-même. C'est-à-dire que cette malléabilité du visage, dans le vif et dans la figuration, permet à l'individu de façonner son image. En ce sens, le phénomène de la chirurgie du visage actualise la notion de masque qui se retrouve dans toutes les cultures et notamment dans le théâtre antique, où il personnifie un caractère ou un rôle. De nos jours, le visage lui-même se conçoit en tant que masque, sans qu'il y ait dissimulation d'un vrai

24. Ces prouesses techno-médicales ont été largement diffusées dans la presse: voir Sabine de la Brosse, «Isabelle, la femme aux deux visages », Paris Match, n 2951, du 8 au 14 décembre 2005, p. 44-52; Le Figaro, «Sciences \& Médecines », 17 avril 2006; de même que sur le réseau Internet. 
visage, mais plutôt élaboration d'une persona. Une telle conception du visage participe de "l'identité performative », selon la notion développée par Judith Butler ${ }^{25}$. La version plastique et artificielle du visage se traduit notamment dans l'autoportrait de l'artiste Ron Mueck, Masque (1997), qui figure exactement les traits de son auteur sous la forme d'un énorme masque (158 x 153 x $124 \mathrm{~cm})$ suspendu par deux câbles dans l'espace de la galerie (fig. 3). Bernadette Wegenstein, dans Getting Under the Skin, analyse plusieurs pages publicitaires du domaine des cosmétiques ${ }^{26}$. Elle note que la tendance à souligner le contour du visage d'un trait suggérant un masque épidermique témoigne de la compétition que mène l'industrie des cosmétiques à l'endroit de la chirurgie esthétique. En ce qui a trait aux enjeux identitaires du visage «à la carte», rappelons que la greffe du visage était réalisable depuis déjà une dizaine d'années, mais qu'aucun hôpital ne voulait jusqu'à maintenant supporter les risques d'un rejet physiologique et les conséquences psychologiques d'un tel bouleversement de l'apparence et par le fait même, de l'identité ${ }^{7}$. Le portrait et le visage relèvent d'une identité en termes de «quoi », selon l'expression de Paul Ricœur dans sa conception dialectique de l'identité personnelle. Or, cette identité est fragile et pose des problèmes, précise Ricœur, car les attributs identitaires sont instables. Ils sont arbitraires et caducs, alors que le sentiment de soi nécessite la permanence ${ }^{28}$. Par conséquent, dans les conditions technologiques actuelles du corps et de l'image, le paradoxe de l'identité personnelle est exacerbé. Dans l'art du portrait contemporain, ce nouveau contexte suscite de nombreuses réactions. On observe une prolifération de visages hybrides, depuis les portraits composites numériques de Nancy Burson au début des années 1980 jusqu'à l'essor actuel du portrait cyberréaliste ${ }^{29}$, en plus de l'exploration de l'identité génétique comme chez Gary Schneider (Genetic SelfPortrait, 1997) et Marc Quinn (Sir John Sulston: A Genomic Portrait, 2001).

25. Judith Butler, Gender Trouble. Feminism and the Subversion of Identity, New York, Routledge Press, 1990; Excitable Speech. A Politics of Performative, New York, Routledge Press, 1997.

26. Bernadette Wegenstein, Getting Under the Skin. Body and Media Theory, Cambridge, Massachusetts, MIT Press, 2006.

27. Le phénomène de la greffe du visage constitue le sujet du film de Georges Franju, Les yeux sans visage (1959). Ce film, qui préfigure de façon romanesque les opérations actuelles dans leurs dimensions médicales et psychologiques, souligne aussi la détresse de l'individu privé de visage et, de là, la nécessité du visage.

28. Paul Ricœur, Soi-même comme un autre, Paris, Éditions du Seuil, 1990.

29. Sur le portrait contemporain, voir Hélène Samson, Du portrait photographique à la fin du $\mathrm{XX}^{e}$ siècle: retour sur le portrait d'identité, [thèse de doctorat], Département d'histoire de l'art et d'études cinématographiques, Université de Montréal, décembre 2005. 
AUTOUR DU PORTRAIT D’IDENTITÉ

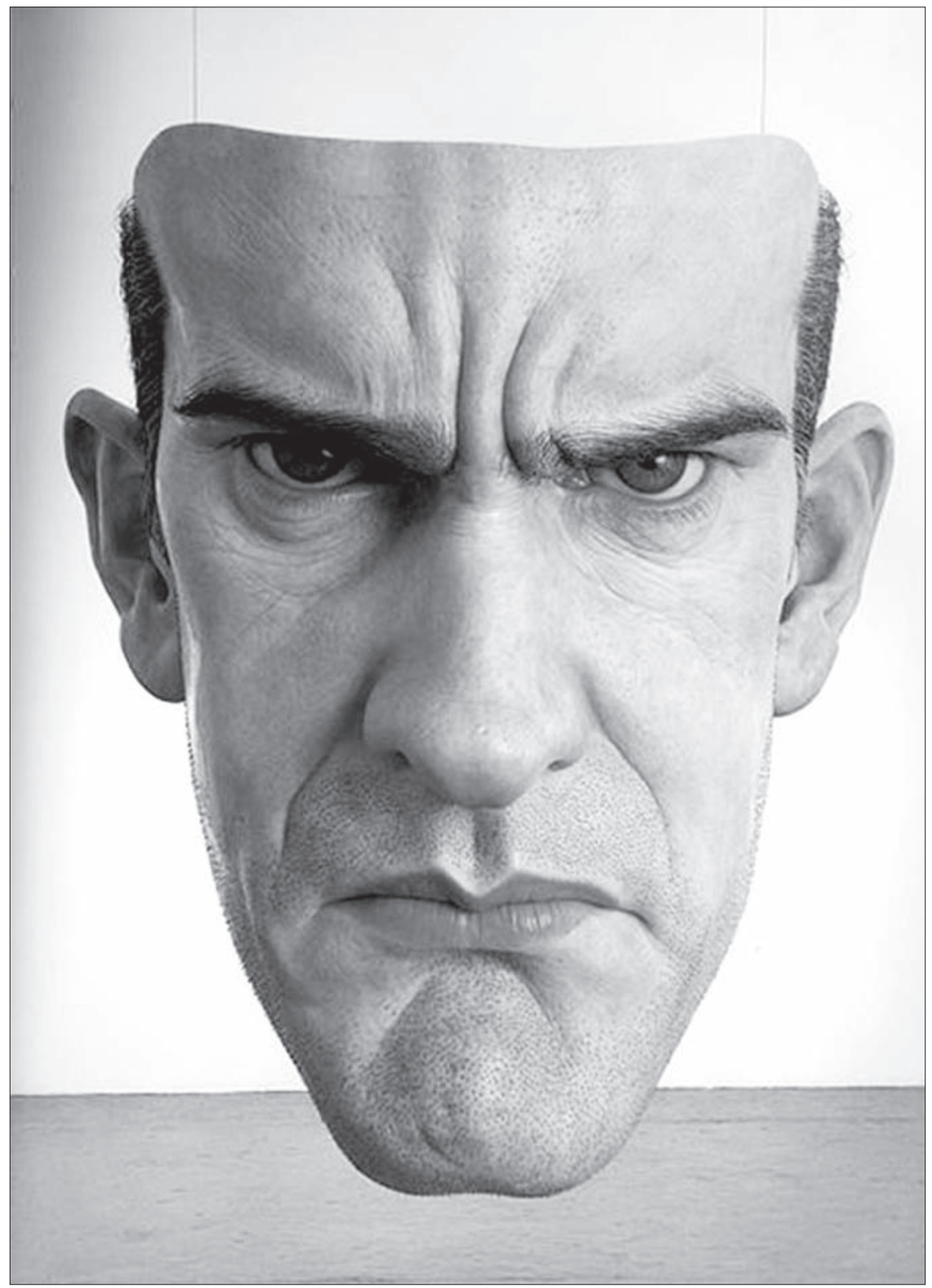

Fig. 3. Ron Mueck, Masque, 1997, résine polyester et technique mixte, 158 x 153 x 124 cm. (c) Ron Mueck. Avec l'aimable autorisation de la Galerie Anthony d'Offay, Londres. 
Les pratiques d'identification que nous avons analysées présentent deux aspects - l'un historique et l'autre phénoménologique - reliés aux mêmes conditions technologiques. D'abord, nous avons constaté le maintien du paradigme du portrait d'identité malgré le changement des modalités d'identification, ce qui suggère une grande stabilité des présupposés modernes que sont l'objectivité et l'inscription corporelle de l'identité. Par ailleurs, au cours de l'évolution des marqueurs de l'identité - du visage à l'ADN - le rôle subsidiaire du visage s'est confirmé. Depuis l'anthropométrie judiciaire de Bertillon, le visage joue un rôle secondaire dans le contrôle des identités - on se rappellera que ce dernier donnait la priorité à l'ossature. Cette dépréciation du visage participe d'un déplacement de la notion d'individualité vers des indices localisés à l'intérieur du corps, comme l'ADN, ou encore vers des indices accessibles par des 82 moyens technologiques qui ne sont pas à la portée de tous. Comme ces indices corporels sont codifiés, l'identité numérique devient centrale dans le système d'identification. En parallèle, nous avons souligné une réelle déstabilisation de la conception du visage dans le phénomène de l'identité personnelle. En effet, la notion et la pragmatique du visage se sont subtilement mais assurément transformées à travers les manipulations numériques et chirurgicales. Nous constatons, à travers l'art contemporain notamment, que la notion de visage en tant que fragment manipulable se développe et que, si le visage joue un rôle subsidiaire dans l'identification, il apparaît comme un artifice privilégié de la performativité de l'identité personnelle. 